\title{
Hybrid P300-Based Brain-Computer Interface to Improve Usability for People With Severe Motor Disability: Electromyographic Signals for Error Correction During a Spelling Task
}

\author{
Angela Riccio, PhD, ${ }^{a, b}$ Elisa Mira Holz, MSc, ${ }^{c}$ Pietro Aricò, PhD, ${ }^{d}$ Francesco Leotta, PhD, ${ }^{d}$ \\ Fabio Aloise, PhD, ${ }^{d}$ Lorenzo Desideri, $\mathrm{MS}{ }^{e}$ Matteo Rimondini, $\mathrm{MS}^{\mathrm{e}}{ }^{\mathrm{e}}$ Andrea Kübler, $\mathrm{PhD},{ }^{\mathrm{c}}$ \\ Donatella Mattia, PhD, ${ }^{a}$ Febo Cincotti, PhD ${ }^{a, d}$ \\ From the ${ }^{a}$ Neuroelectrical Imaging and BCI Lab, Fondazione Santa Lucia, Rome, Italy; ${ }^{b}$ Department of Psychology, Sapienza University of \\ Rome, Rome, Italy; ${ }^{C}$ Institute of Psychology, University of Würzburg, Würzburg, Germany; ${ }^{d}$ Department of Computer, Control, and Management \\ Engineering Antonio Ruberti, Sapienza University of Rome, Rome, Italy; and ${ }^{e}$ Ausilioteca, AIAS Bologna Onlus, Bologna, Italy.
}

\begin{abstract}
Objective: To evaluate the impact of a hybrid control on usability of a P300-based brain-computer interface (BCI) system that was designed to control an assistive technology software and was integrated with an electromyographic channel for error correction.

Design: Proof-of-principle study with a convenience sample.

Setting: Neurologic rehabilitation hospital.

Participants: Participants $(\mathrm{N}=11)$ in this pilot study included healthy $(\mathrm{n}=8)$ and severely motor impaired $(\mathrm{n}=3)$ persons. The 3 people with severe motor disability were identified as potential candidates to benefit from the proposed hybrid BCI system for communication and environmental interaction.

Interventions: To eventually investigate the improvement in usability, we compared 2 modalities of BCI system control: a P300-based and a hybrid P300 electromyographic-based mode of control.

Main Outcome Measures: System usability was evaluated according to the following outcome measures within 3 domains: (1) effectiveness (overall system accuracy and P300-based BCI accuracy); (2) efficiency (throughput time and users' workload); and (3) satisfaction (users' satisfaction). We also considered the information transfer rate and time for selection.

Results: Findings obtained in healthy participants were in favor of a higher usability of the hybrid control as compared with the nonhybrid. A similar trend was indicated by the observational results gathered from each of the 3 potential end-users.

Conclusions: The proposed hybrid BCI control modality could provide end-users with severe motor disability with an option to exploit some residual muscular activity, which could not be fully reliable for properly controlling an assistive technology device. The findings reported in this pilot study encourage the implementation of a clinical trial involving a large cohort of end-users.

Archives of Physical Medicine and Rehabilitation 2015;96(3 Suppl 1):S54-61

(C) 2015 by the American Congress of Rehabilitation Medicine

Presented to the National Institutes of Health, National Science Foundation, and other organizations (for a full list, see http://bcimeeting.org/2013/sponsors.html), June 3-7, 2013, Asiloma Conference Grounds, Pacific Grove, CA.

Supported by the European ICT Programme Project FP7-224631 (Tools for Brain-Computer Interaction). This paper only reflects the authors' views and funding agencies are not liable for any use that may be made of the information contained herein.

Disclosures: none.

In recent years, the development of communication systems based on brain-computer interface (BCI) technology has begun to take advantage of the assistive technology (AT) design principles ${ }^{1}$ such as the user-centered design (UCD). ${ }^{2}$ By adopting this approach, the research priority would be readdressed from the BCI engineering design to the end-users' needs. Thus, the BCI may be foreseen also as an additional channel among others to access AT devices.
\end{abstract}


In the course of some progressive neurodegenerative diseases, before the complete locked-in state occurs, the residual muscle activity may still be exploitable. This may also hold true for other neurologic diseases: for instance, in the various rehabilitation phases of acquired brain injury, patients may recover minimal muscular activity; in paralyses caused by congenital brain damage (ie, cerebral palsy), residual movements may be maintained. In several cases, such residual motor abilities would not be functionally valid to fully and/or continuously control an ordinary AT (eg, because of weakness, fatigability, spasticity, or dystonia). On the other hand, residual muscular activity may function as one of the input channels of a hybrid BCI, which can be defined as a combination of different signals including at least $1 \mathrm{BCI}$ channel. It could be a combination of (1) 2 BCI channels or (2) BCI and other biosignals (such as electromyographic [EMG] activity) or special AT input devices (eg, joysticks, switches). ${ }^{3}$

In a previous study, ${ }^{4}$ a BCI based on the P300 event-related potential (for a review, see Kleih et $\mathrm{al}^{5}$ ) was combined with an AT software for communication and environmental control. The P300-based BCI (P300-BCI) merged into the AT software was considered as an additional but exclusive control option. Such a BCI-based system was successfully tested with severely disabled potential end-users, and system evaluation exploited metrics inspired by AT principles. ${ }^{6}$ Following the UCD ${ }^{7}$ the end-users' feedback was collected on the usability of such an implemented system and was addressed by endowing the system with a hybrid control. ${ }^{8}$ The hybrid control consisted of integrating an additional channel to subserve the function of deleting incorrect BCI selections that exploited the EMG signals generated by the end-user's residual muscular activity.

The ultimate aim of this feasibility study was to investigate the expected improvement in the usability of the P300-BCI system after the integration of the (EMG) hybrid control. For this purpose, we adopted an experimental design consisting of a direct comparison of the systems with and without the hybrid input used by a convenience sample of healthy and disabled participants. For evaluation purposes, we adopted the framework of metrics previously applied by Zickler et $\mathrm{al}^{6}$ that was adapted to take into account the hybrid component of the system control in the evaluation procedure. Accordingly, the expected improvement in the usability was assessed in terms of system effectiveness (expressed as system accuracy and BCI accuracy), efficiency (expressed as the perceived user workload and the time to output a symbol, defined as the throughput time), and the satisfaction expressed by the participants using the system. The information transfer rate (ITR) ${ }^{9}$ and the time for selection were also considered as measures reflecting both the efficiency and effectiveness domains. We expected differences in the measures between a hybrid and nonhybrid system usage toward an improvement for the hybrid mode, in the 3 usability domains. Our preliminary findings indicated a

\begin{tabular}{|c|c|}
\hline \multicolumn{2}{|c|}{ List of abbreviations: } \\
\hline AT & assistive technology \\
\hline BCI & brain-computer interface \\
\hline EEG & electroencephalogram \\
\hline EMG & electromyographic \\
\hline ITR & information transfer rate \\
\hline NASA-TLX & $\begin{array}{l}\text { National Aeronautics and Space Administration-Task } \\
\text { Load Index }\end{array}$ \\
\hline P300-BCI & P300-based brain-computer interface \\
\hline
\end{tabular}

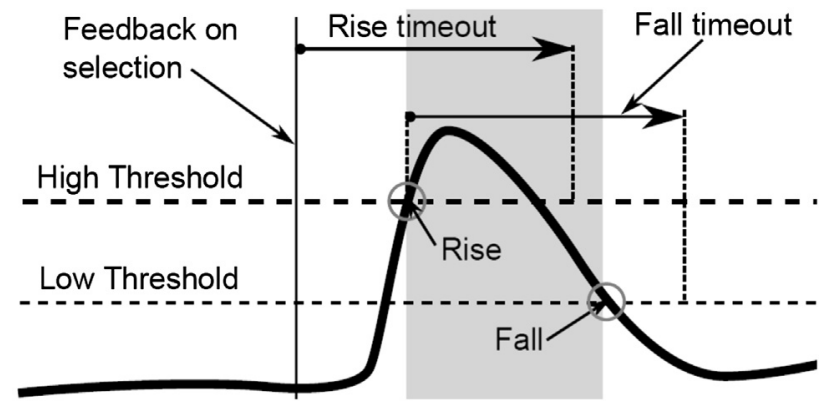

Fig 1 EMG signal onset (exceeding the high threshold) must occur within a specified time window after the feedback (rise timeout). EMG signal offset (exceeding the low threshold) must occur within a specified time window after the EMG signal onset (fall timeout).

higher usability of the hybrid control to accomplish a spelling task, and also revealed that the evaluation needs to be multifaceted.

\section{Methods}

\section{Hybrid system}

To pursue adherence to UCD, the hybrid system was conceptualized to adapt to several degrees of residual motor activity. EMG activity classification was used to cancel a speller command previously issued through the P300-BCI. To reduce the occurrence of false positives, the EMG activity classification process was enabled only within a certain time window after the presentation of the feedback of the P300 classification. Constraints aimed at discarding muscular activity not intentionally related to the user's need to cancel a wrong P300-BCI selection were introduced, as described in figure 1.

The AT software was QualiWorld ${ }^{\mathrm{a}}(\mathrm{QW})$. The visual stimulation of the P300-BCI was overlaid on top of the QualiWorld window (see Riccio et $\mathrm{al}^{8}$ for details).

\section{Participants}

Eight healthy participants ( 3 women; mean age \pm SD, $28 \pm 7 y$ ) and 3 end-users with severe motor impairment participated in the evaluation of the hybrid P300-QW prototype. Table 1 illustrates the end-users' demographic and clinical characteristics. They all had a severe motor disability associated with an impairment of their communication capacity.

Seven participants (5 healthy participants and 2 end-users [end-users 2 and 3 in table 1]) of the 11 were naïve to BCI protocol and training.

The study was approved by the ethical committee of Fondazione Santa Lucia, IRCCS Rome, Italy. All participants (or legal guardian, when needed) gave their written informed consent for participation in the study.

\section{Experimental protocol}

\section{Screening session}

During the first session (screening session), participants were screened with 4 types of visual stimuli to elicit the P300 components that served best for the online BCI paradigm. Visual stimuli varied in colors (red vs green) and shapes (dot vs grid) as 
Table 1 Demographic and clinical characteristic of the 3 end-users involved in the study

\begin{tabular}{llllll}
\hline $\begin{array}{l}\text { End- } \\
\text { User No. }\end{array}$ & $\begin{array}{l}\text { Age } \\
\text { (y) }\end{array}$ & Sex & Diagnosis & $\begin{array}{l}\text { Barthel Index } \\
(0-100)^{14}\end{array}$ & Description \\
\hline 1 & 48 & F & Brainstem stroke & 0 & $\begin{array}{c}\text { Severe tetraparesis (residual movement of right arm, gravity eliminated), } \\
\text { wheelchair, head movement, facial mimic, no speech } \\
\text { Hemiplegic, wheelchair, dysarthria }\end{array}$ \\
3 & 54 & M & $\begin{array}{c}\text { Hemorrhagic } \\
\text { stroke }\end{array}$ & 35 & Tetraparesis, wheelchair, dysarthria \\
\hline
\end{tabular}

Abbreviations: $F$, female; $M$, male.

shown in figure 2. An offline cross-validation was performed to identify the most effective stimulation and the number of stimulation sequences to be delivered to each participant. The minimum number of stimulation sequences needed to reach the $80 \%$ of accuracy estimated in the offline cross-validation was then set for the subsequent online session. This allowed us to expect a spelling error to occur every 5 selections in the online session. The number of stimulation sequences ranged between 2 (4 stimulations) and 10 (20 stimulations).

In the group of healthy participants, the EMG activity was always detected from the extensor carpi radialis longus muscle, activated by executing a wrist extension. The end-users' target muscle was identified on the basis of their residual functional voluntary movements, to individually customize the EMG signaldriven control channel. End-user 1 performed a slight extension of the wrist detected by placing the electrode over the flexor carpi radialis; for end-user 2, wrist extension was identified as the most comfortable movement, and the EMG activity was recorded from the extensor carpi radialis longus. End-user 3 performed an incomplete flexion of the fingers (slightly clenching the fist), and the electrode was placed over the flexor digitorum superficialis.

For each participant, the (1) onset and the (2) offset threshold of the EMG signal amplitude and the (3) time window after the
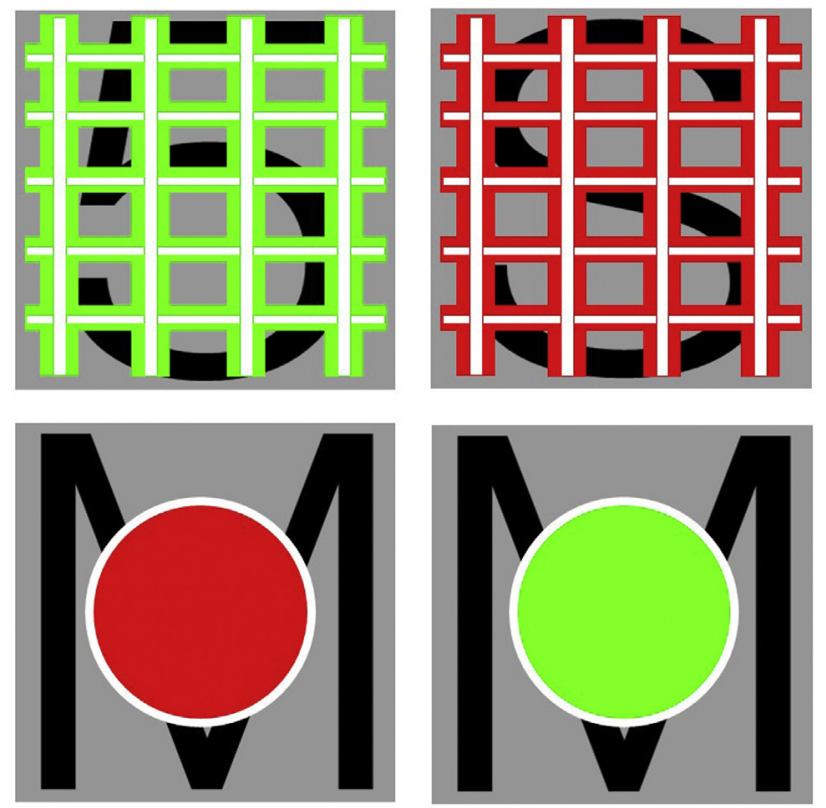

Fig 2 Visual stimuli to elicit the P300 components presented to participants in the first session. feedback (within which the EMG signal onset and offset must occur in order to delete a wrong P300-BCI command) were defined (see fig 1). These thresholds were manually set by the operator, after the participants performed the required movement in a natural way (see also the Electrophysiological Recording section for details).

\section{BCI session}

During the second session (BCI session), participants were asked to spell 3 predefined words (respectively of 5, 8 , and 8 letters each) using the P300-BCI speller. Deletion of incorrect selections differed in the 2 conditions:

1. Nonhybrid condition: By means of the P300-BCI itself, choosing a backspace command on the QualiWorld virtual keyboard.

2. Hybrid condition: By means of the EMG signal-driven control; in case of a missed deletion, a BCI backspace had to be used as in condition 1.

The sequence of conditions was randomized.

All questionnaires (see Evaluation Parameters section) were administered at the end of each experimental condition.

\section{Evaluation parameters}

The hybrid and nonhybrid control conditions were compared in terms of usability, as defined in the ISO $9241-210 .^{7}$ As in the articles by Riccio ${ }^{4}$ and Zickler $^{6}$ and colleagues, the 2 conditions were compared within 3 usability domains: effectiveness, efficiency, and satisfaction. Some adaptations of the previously reported framework of metrics were, however, introduced in this study because of the specificities of the hybrid control and the experimental design. In this regard, effectiveness was quantified by the system accuracy, considering the BCI system as in the article by Mason and Birch. ${ }^{10}$ On the basis of the cited study, ${ }^{10}$ a selection was considered the output of the whole system that included the P300 selection and EMG signal-driven correction. Hence, the block composed of the (wrong) selection, the EMG signal-driven deletion (which cancels the previous selection), and the following (correct) selection were considered as a single output issued by the hybrid system (fig $3 \mathrm{~A}$ ). In the nonhybrid condition, a wrong selection had to be canceled by means of the $\mathrm{BCI}$, and thus it was followed by a stimulation trial leading to the selection of the backspace key. This latter selection was in turn followed by another selection, namely the correct letter (fig 3B). In this case, the 3 actions were considered separately as 3 (2 correct, 1 wrong) different outputs of the system. The system 


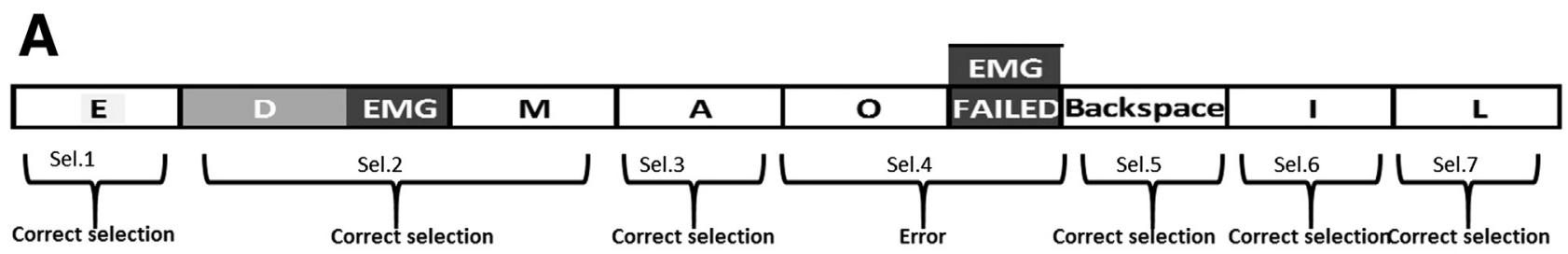

B

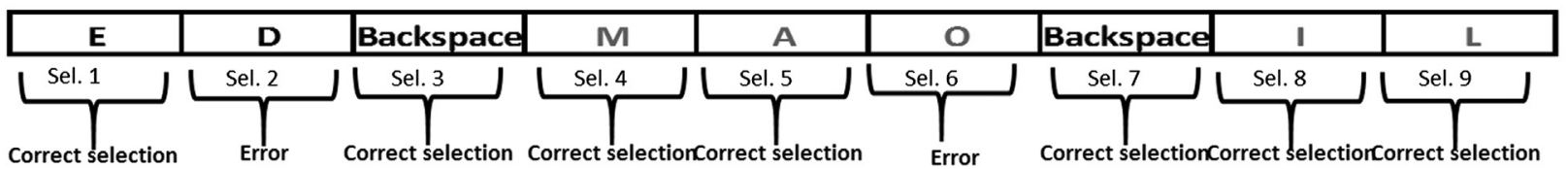

Fig 3 Concept of correct selection and error in the hybrid system (A) and nonhybrid system (B). (A) A selection (Sel.) is considered the output of the system. For example, Sel.2 (which corresponds to the output of the system) is considered the (wrong) selection (letter D) annulled by means of EMG activity together with the right selection (letter M). (B) The (wrong) selection is considered per se an output of the system, because it needs one more BCI output (Backspace) to be deleted.

accuracy (reported in terms of percentage of correct outputs) was then calculated by dividing the number of correct outputs by the number of total outputs.

In addition, in order to elucidate to what extent the presence of a double control channel (electroencephalogram [EEG] and EMG activity) would have influenced the exclusive P300-based classification, we also estimated the accuracy of the P300-based BCI selections achieved by the participants under the hybrid condition. This BCI accuracy was then calculated as the ratio between the number of P300-BCI correct selections and the total number of P300-BCI selections (no EMG signal-driven error correction was considered) and reported in terms of percentage of correct selections.

Efficiency was measured in terms of throughput time and user workload. Here, we defined the throughput time as the total time to accomplish the task divided by the total number of (correct + wrong) outputs. Thus, it provides a measure of the time (in seconds) needed to receive any output from the system as a whole. As in previous studies, ${ }^{4,6}$ the user's perceived workload was measured by means of the National Aeronautics and Space AdministrationTask Load Index (NASA-TLX), ${ }^{11}$ with scores ranging from 0 to 33.3 for each factor (mental demand, physical demand, temporal demand, performance, effort, frustration) and from 0 to 100 for the total workload scores.

Finally, satisfaction was reported by the participants by means of a visual analog scale on which they were asked to indicate the level of satisfaction experienced with the system, ${ }^{6}$ ranging from 0 (not satisfied at all) to 10 (completely satisfied).

The ITR as defined by Wolpaw et $\mathrm{al}^{9}$ (bits $\times$ minutes) was also used in this context. We considered the ITR belonging to both the effectiveness and efficiency domains, since this metric is sensitive to both usability domains. Similar to the ITR, the time for selection was considered to span both the whole system effectiveness and efficiency. The time for selection (in seconds) was referred to as the ratio between the total time required to successfully complete the task (to spell a given word) and the minimum number of selections needed to execute it (number of letters composing the word to be spelled).

Because of the different lengths of the words, the total time spent in accomplishing the spelling of each word could not be averaged; thus, each total time was divided by the number of letters contained in each word. The resulting time for selection also represented the mean time needed to write a correct symbol.

\section{Electrophysiological recording}

EEG and EMG signals were amplified and digitized by a 16channel biosignal amplifier (gUSBAmp ${ }^{\mathrm{b}}$ ), and the sampling rate was at $256 \mathrm{~Hz}$. Data were acquired from 8 monopolar EEG channels (Fz, Cz, Pz, Oz, P3, P4, PO7, PO8) referenced at the right earlobe and grounded at the left mastoid, and 1 bipolar EMG channel mounted on the skin above the target muscle.

\section{EEG and EMG signal-driven classification}

Target and nontarget EEG-evoked responses were discriminated by means of a linear classifier, whose optimal weights were set in an offline analysis (calibration) by means of a stepwise linear discriminant analysis. ${ }^{12}$ The number of maximum iterations of the algorithm was set to 60 . For each iteration, features with a $P$ value $<0.1$ and those with a $P$ value $>.15$ were added to or removed from the model, respectively. The epochs were down-sampled, replacing each segment of 3 samples with their mean value and then reducing an epoch to 68 samples. The resulting $8 \times 68$ data arrays were concatenated, creating a 544-element features vector $\mathrm{v}_{\mathrm{f}}$ for each stimulus.

Real-time extraction of the linear envelope ${ }^{13}$ was used to obtain an EMG feature that was directly correlated with the temporal profile of contraction strength. Once the linear envelope was computed, the thresholds (defined during the screening session) were used to detect the presence of intentional muscular contraction (see fig 1).

\section{Data analysis}

Data recorded from the healthy participants and end-users were analyzed separately. In the group of healthy participants, any difference between the 2 conditions was explored by means of a nonparametric Wilcoxon matched-pairs test applied for each of the outcome measures. The data set belonging to each end-user was analyzed qualitatively with reference to the results obtained from the group of healthy participants. 

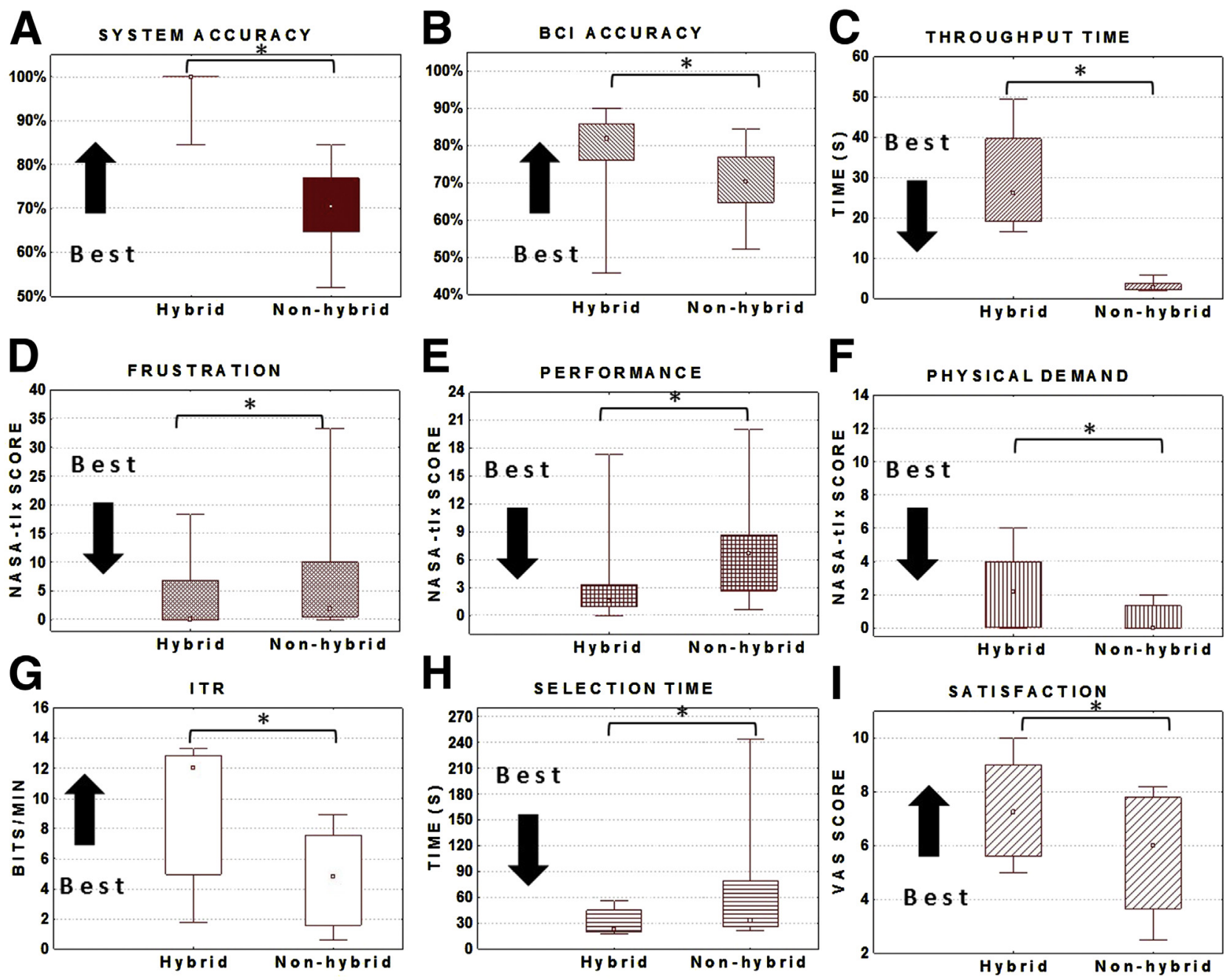

Median 25\%-75\% Min-Max

Fig 4 Boxplots illustrate the variables that showed a significant difference between the hybrid system (left) and nonhybrid system (right) in healthy participants. Asterisks show significance. (A) System accuracy. (B) BCI accuracy. (C) Throughput time. (D) Frustration. (E) Performance. (F) Physical demand. (G) ITR. (H) Selection time. (I) Satisfaction. Boxplots illustrate medians with 25th to 75th percentiles, with lines extending to 10th and 90th percentiles. Abbreviations: Max, maximum; Min, minimum.

\section{Results}

\section{Healthy participants}

\section{Effectiveness}

The system accuracy was significantly higher for the hybrid condition (median, 100\%; range, $84.5 \%-100 \%$ ) with respect to the nonhybrid condition (median, $70.3 \%$; range, $52 \%-84.5 \%$; $\mathrm{Z}=2.5 ; P<.05)$ (fig $4 \mathrm{~A}$ ).

The BCI exhibited a significantly higher accuracy (BCI accuracy) in the hybrid condition (median, 81.9\%; range, $45.7 \%-$ $90 \%$ ) than in the nonhybrid condition (median, $70.3 \%$; range, $52 \%-84.5 \% ; \mathrm{Z}=2.5 ; P<.05$ ) (fig $4 \mathrm{~B}$ ).

The average of all EMG signal-driven actions performed to correct errors was 2.22 (range, 2-14 total selections for participant). For all the healthy participants, the EMG activity classification accuracy was $100 \%$.

\section{Efficiency}

The throughput time was significantly shorter in the nonhybrid condition (median, 2.6s; range, 2-5.9s) than in the hybrid condition (median, 26.3s; range, 16.5-49.4s; $\mathrm{Z}=2.5 ; \mathrm{P}<.001$ ) (fig 4C). The level of frustration perceived by the healthy users was significantly lower for the hybrid (median, .01; range, 0-18.3) as compared with the nonhybrid condition (median, 1.8; range, $0-33.3 ; \mathrm{Z}=2.2$; $P<.01$ ) (fig 4D). Similar results were obtained for the perceived performance, a factor influencing workload construction (hybrid: median, 1.5; range, 0-17.3; nonhybrid: median, 6.7; range, 0.6-20; $\mathrm{Z}=2.5 ; P<.01$ ) (fig $4 \mathrm{E})$. A significant difference was also found for the physical demand, with higher scores for the hybrid condition (median, 2.2; range, $0-6$ ) as compared with the nonhybrid condition (median, 0 ; range, $0-2 ; \mathrm{Z}=2 ; P<.05$ ) (fig $4 \mathrm{~F}$ ). No significant differences between the 2 conditions were found for the remaining 3 factors of the NASA-TLX scale (mental demand: $Z=-0.4, P=0.6$; temporal demand: $\mathrm{Z}=0.1, P=0.9$; effort: $\mathrm{Z}=1.2, P=0.2$ ) and for the total workload scores $(\mathrm{Z}=1.5, P=0.1)$. 
Table 2 Summary overview of the metrics and results (healthy subjects)

\begin{tabular}{llcccc}
\hline Usability Domains & Metrics & Median Hybrid & Median Nonhybrid & Range Hybrid & Range Nonhybrid \\
\hline Effectiveness & BCI accuracy (\%) & 81.9 & 70.3 & $45.7-90$ & $52-84.5$ \\
Efficiency & System accuracy (\%) & 100 & 70.3 & $84.5-100$ & $52-84.5$ \\
& Throughput time (s) & 26.3 & 2.6 & $16.5-49.4$ & $2-5.9$ \\
& Mental demand (NASA-TLX) & 13.3 & 14 & $0.3-26.7$ & $0.3-25$ \\
& Physical demand (NASA-TLX) & 2.2 & 0 & $0-6$ & $0-2$ \\
& Temporal demand (NASA-TLX) & 8.5 & 7 & $0.3-21.3$ & $0.02-19$ \\
& Performance (NASA-TLX) & 1.5 & 6.7 & $0-17.3$ & $0.6-20$ \\
& Effort (NASA-TLX) & 5.3 & 10 & $0.1-19$ & $0.02-20$ \\
Satisfaction & Frustration (NASA-TLX) & 0.01 & 1.8 & $0-18.3$ & $0-33.3$ \\
Effectiveness/efficiency & Total workload (NASA-TLX) & 48.8 & 52.3 & $5.6-71.3$ & $7-99$ \\
& Satisfaction VAS & 7.2 & 6 & $5-10$ & $2.5-8.2$ \\
& ITR (bits x min) & 12 & 4.7 & $1.8-13.3$ & $0.6-8.9$ \\
Abbreviation: VAS, visual analog scale. & Time for selection (s) & & 33.4 & $18-56$ & $21.5-243.5$ \\
\hline
\end{tabular}

\section{Metrics in between effectiveness and efficiency}

The ITR was significantly higher in the hybrid (median, 12 bits $\times$ min; range, $1.8-13.3$ bits $\times$ min) as compared with the nonhybrid condition (median, 4.7 bits $\times$ min; range, $0.6-8.9$ bits $\times$ min; $\mathrm{Z}=2.5 ; P<.01$ ) (fig $4 \mathrm{G}$ ). Furthermore, the time for selection was significantly shorter for the hybrid condition (median, 22.5s; range, 18-56s) than for the nonhybrid condition (median, 33.4s; range, $21.5-243.5 \mathrm{~s} ; \mathrm{Z}=2.5 ; P<.01$ ) (fig $4 \mathrm{H})$.

\section{Satisfaction}

The healthy group scored a significantly higher level of satisfaction while using the hybrid version of the system (hybrid condition: median, 7.2; range, 5-10; nonhybrid condition: median, 6; range, $2.5-8.2 ; \mathrm{Z}=2.4 ; P<.01$ ) (fig $4 \mathrm{I}$ ).

For a summary overview of the metrics and results, see table 2 .

\section{End-users}

The qualitative analysis conducted with the data set obtained from the 3 end-users revealed a trend toward a higher usability when the system was used in the hybrid as compared with the nonhybrid mode (fig 5). For all end-users, the system and BCI accuracy (fig 5A and B), the throughput time (fig 5C), the ITR (fig $5 \mathrm{G}$ ), the time for selection (fig $5 \mathrm{H}$ ), and the satisfaction scores (fig 5I) displayed a trend similar to what was observed in the healthy participants.

When considering the level of perceived workload, a certain degree of variability was found between the 3 end-users. Similar to healthy participants, all end-users scored performance (as a factor of the NASA-TLX) as lower in the hybrid condition (fig $5 \mathrm{E}$ ). The perceived level of frustration (fig 5D) was variable, as it was scored as higher for the hybrid condition by end-users 1 and 2 , and lower by end-user 3 . The hybrid control was found to be associated with a higher level of physical demand by end-users 2 and 3 (similar to healthy participants), but not by end-user 1 (fig 5F).

Finally, the end-users performed, 8 deletions (end-user 1), 6 deletions (end-user 2), and 1 deletion (end-user 3) by means of EMG activity. Only end-user 1 missed the deletion with EMG activity 3 times.

\section{Discussion}

The evaluation with healthy participants partly supported the initial hypothesis that the integration of the EMG channel into the system would lead to an improvement of the overall system usability. In terms of effectiveness, the advantage of the hybrid system was clearly shown by the significant increase in the system accuracy. Conversely, the efficiency of the hybrid system appeared lower with respect to the exclusive BCI control modality as expressed by a longer throughput time, which depends, however, on the total number of system outputs that is lower in the hybrid modality.

The decrease of this efficiency measure was balanced by a superiority of the hybrid system in terms of time for selection and ITR, which accounts for both the accuracy and the resources spent by the users in controlling the system. In particular, despite a longer time to produce an output (longer throughput time) that is required with the hybrid mode, the EMG signal-based correction leads to a sensible increase in the accuracy of outputs (system accuracy), thus resulting in a decrease in the time needed to accomplish a task (ie, time for selection).

A significant decrease in the users' workload was also observed, particularly in terms of frustration and performance perceived. The physical demand was rated higher in the hybrid condition than in the nonhybrid condition. This was probably due to the characteristics of this control modality requiring a major physical involvement.

Noticeably, the presence of an additional (EMG) channel to control the system did not negatively influence the users' performance in terms of pure BCI-based control despite being challenged with a "dual task." Nevertheless, this dual task did not burden the mental demand factor whose scores did not show a significant difference between the 2 conditions.

Finally, the healthy participants expressed an overall higher satisfaction with the hybrid system as compared with the nonhybrid system.

The similarity in system usage performance showed by the 3 end-users corroborates the added value of the hybrid control concept.

The system-related usability metrics (ie, system accuracy, ITR, time for selection) showed for all end-users a trend in line with 

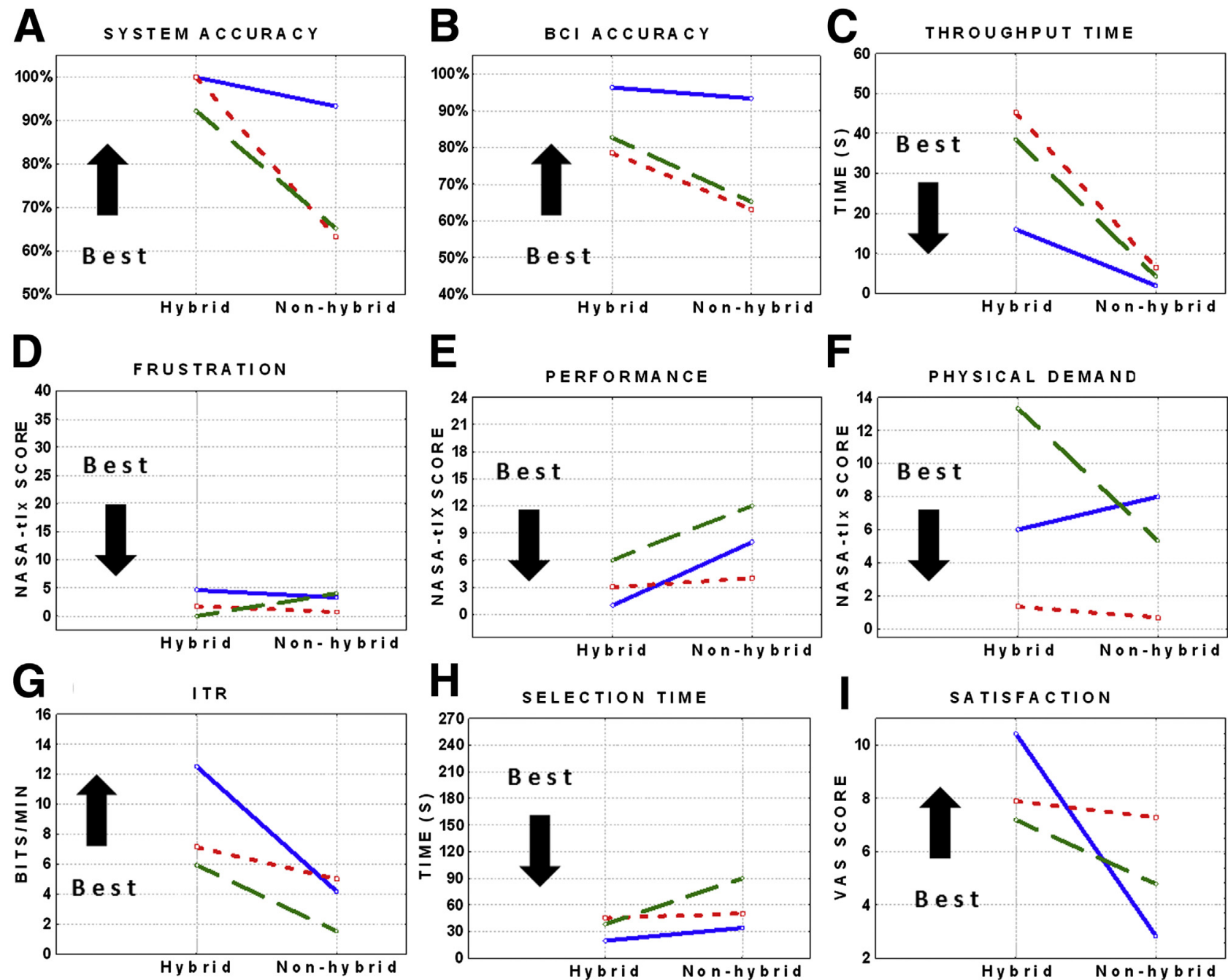

Case 1

Hybrid

Hy b rid

Non-hy brid

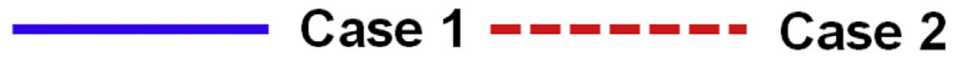

Fig 5 Line plots illustrate the scores observed with the hybrid system (left) and nonhybrid system (right) in the 3 end-users for the same variables shown in figure 4 (scores of the healthy participants). (A) System accuracy. (B) BCI accuracy. (C) Throughput time. (D) Frustration. (E) Performance. (F) Physical demand. (G) ITR. (H) Selection time. (I) Satisfaction.

what observed in the healthy group - that is, in favor of the hybrid mode of control. In addition, all end-users scored their satisfaction as higher when dealing with the hybrid compared with the nonhybrid control.

In regard to the efficiency metrics, the throughput time displayed a trend similar to what was observed in the healthy participants. Differently, the improvement of the hybrid mode efficiency was not consistent between the end-users as revealed by the NASA-TLX self-reported factor scores (see fig 5). This variability in the self-reported usability metrics between end-users may reflect that the end-users were not uniform in terms of their physical disabilities and etiology of their disabilities. The perception of the system usability is likely influenced by the specific physical disability of the end-user, given the hybrid nature of the proposed solution. These overall observational findings corroborate the importance of adopting a user-centered approach to fulfill the needs of end-users with various physical disabilities. ${ }^{14-17}$ They also encourage further validation of such hybrid access to AT devices with a large cohort of potential end-users.

Moreover, the improvement of usability after the introduction of the hybrid channel consolidates the adoption of the usercentered approach in the lifecycle of BCI system development. The hybrid concept was indeed implemented as the consequence of users' feedback reported in Zickler's study, ${ }^{6}$ wherein the purely BCI-based system was evaluated by potential end-users, and they clearly suggested "an interface that integrates BCI with other input channels." ${ }^{6(\mathrm{p} 243)}$ Although it remains to be validated in a larger sample of potential end-users, we are confident that this implementation would increase the "functionality" and "easiness to use" of a BCI system, 2 factors mentioned by end-users as highly relevant in BCI future design. ${ }^{18}$

Finally, it is important to outline one aspect that emerges from this pilot study and goes beyond the specific testing of the 
proposed hybrid system. The evaluation results reported here in healthy users confirm the suitability of the usability framework of metrics originally presented in the studies by Zickler $^{6}$ and Riccio ${ }^{6}$ to evaluate newly proposed BCI applications of different complexity beyond their technical features.

\section{Study limitations}

The presented feasibility study involved healthy participants and 3 end-users. This limited number of participants prevents us from fully evaluating the proposed system. Future studies involving a larger cohort of end-users with different degrees of motor impairment (eg, spasticity, degradation of motor response and general artifact caused by involuntary movements) should be performed to better investigate the potential of this hybrid approach in real-life scenarios.

\section{Conclusions}

A hybrid BCI system (P300-based BCI and EMG signal-driven control channel) for communication was developed to exploit severely disabled end-users' residual muscle activity as one input channel to correct possible errors induced by the BCIbased spelling. Despite the limits imposed by the small sample size, the overall findings of this pilot study supported a certain level of gain in usability for the hybrid BCI. Therefore, the integration of the hybrid channel can be considered a significant step in increasing the functionality and the speed of the system, 2 issues that were rated very important by potential BCI endusers. ${ }^{18}$ The validation of the proposed hybrid BCI approach with a larger cohort of end-users is needed to consolidate these preliminary findings and to return a lower variance in the selfreported metrics. We expected that this would allow for the prediction of those potential candidates who may best benefit from hybrid BCI systems.

\section{Suppliers}

a. QualiLife Inc.

b. g.tec Medical Engineering GmbH.

\section{Keywords}

ALS (amyotrophic lateral sclerosis); Assistive technology; Braincomputer interfaces; Brain injuries; Event-related potentials; P300; Rehabilitation

\section{Corresponding author}

Angela Riccio, PhD, Neuroelectrical Imaging and BCI Lab, Fondazione Santa Lucia Via Ardeatina, 306, I-00179 Roma, Italy. E-mail address: a.riccio@hsantalucia.it.

\section{References}

1. Kaufmann T, Völker S, Gunesch L, Kübler A. Spelling is just a click away-a user-centered brain-computer interface including autocalibration and predictive text entry. Front Neurosci 2012;6:72.

2. Schreuder M, Riccio A, Risetti M, et al. User-centered design in braincomputer interfaces-a case study. Artif Intell Med 2013;59:71-80.

3. Millán JD, Rupp R, Müller-Putz GR, et al. Combining brain-computer interfaces and assistive technologies: state-of-the-art and challenges. Front Neurosci 2010;4:161.

4. Riccio A, Leotta F, Bianchi L, et al. Workload measurement in a communication application operated through a P300-based braincomputer interface. J Neural Eng 2011;8:025028.

5. Kleih SC, Kaufmann T, Zickler C, et al. Out of the frying pan into the fire-the P300-based BCI faces real-world challenges. Prog Brain Res 2011;194:27-46.

6. Zickler C, Riccio A, Leotta F, et al. A brain-computer interface as input channel for a standard assistive technology software. Clin EEG Neurosci 2011;42:236-44.

7. Ergonomics of human-system interaction-part 210: human-centred design for interactive systems: ISO 9241-210. International Organization for Standardization; 2010.

8. Riccio A, Holz EM, Aricò P, et al. A hybrid control of a P300-based BCI: a solution to improve system usability? Proceedings of the Fifth International Brain-Computer Interface Meeting: defining the future; 2013 Jun 3-7; Pacific Grove, CA. Available at: http://dx.doi.org/10. 3217/978-3-85125-260-6-167.

9. Wolpaw JR, Birbaumer N, McFarland DJ, Pfurtscheller G, Vaughan TM. Brain-computer interfaces for communication and control. Clin Neurophysiol 2002;113:767-91.

10. Mason SG, Birch GE. A general framework for brain-computer interface design. IEEE Trans Neural Syst Rehabil Eng 2003;11:70-85.

11. Hart SG. NASA-task load index (NASA-TLX); 20 years later. Proc Hum Factors Ergon Soc Annu Meet 2006;50:904-8.

12. Krusienski DJ, Sellers EW, Cabestaing F, et al. A comparison of classification techniques for the P300 speller. J Neural Eng 2006;3:299-305.

13. Merletti R, Parker P. Electromyography physiology, engineering, and noninvasive applications. Piscataway, Hoboken: IEEE Pr, WileyInterscience; 2004.

14. Mahoney FI, Barthel DW. Functional evaluation: the Barthel Index. Md State Med J 1965;14:61-5.

15. Collinger JL, Boninger ML, Bruns TM, Curley K, Wang W, Weber DJ. Functional priorities, assistive technology, and brain-computer interfaces after spinal cord injury. J Rehabil Res Dev 2013;50:145-60.

16. Blain-Moraes S, Schaff R, Gruis KL, Huggins JE, Wren PA. Barriers to and mediators of brain-computer interface user acceptance: focus group findings. Ergonomics 2012;55:516-25.

17. Huggins JE, Wren PA, Gruis KL. What would brain-computer interface users want? Opinions and priorities of potential users with amyotrophic lateral sclerosis. Amyotroph Lateral Scler 2011;12:318-24.

18. Zickler C, Di donna V, Kaiser V, et al. BCI applications for people with disabilities: defining user needs and user requirements. In: Emiliani PL, Burzagli L, Como A, Gabbanini F, Salminen A-L, editors. Assistive technology from adapted equipment to inclusive environments. Vol 25 Assistive technology research series. Amsterdam: IOS Pr; 2009. p 186-9. 